\title{
Cerebellum and ocular motor control
}

\author{
Amir Kheradmand $^{1 *}$ and David S. Zee $e^{1,2,3,4}$ \\ ${ }^{1}$ Department of Neurology, The Johns Hopkins University School of Medicine, Baltimore, MD, USA \\ ${ }^{2}$ Department of Ophthalmology, The Johns Hopkins University School of Medicine, Baltimore, MD, USA \\ ${ }^{3}$ Department of Otolaryngology-Head and Neck Surgery, The Johns Hopkins University School of Medicine, Baltimore, MD, USA \\ ${ }^{4}$ Department of Neuroscience, The Johns Hopkins University School of Medicine, Baltimore, MD, USA
}

\section{Edited by:}

Sergio Carmona, Instituto de

Neurociencias de Buenos Aires

INEBA, Argentina

Reviewed by:

Sergio Carmona, Instituto de

Neurociencias de Buenos Aires

INEBA, Argentina

Matthew J. Thurtell, University of

lowa, USA

Matt Stewart, Johns Hopkins

Hospital, USA

\section{*Correspondence:}

Amir Kheradmand, Oculomotor Lab, Department of Neurology, The Johns Hopkins Hospital, Path 2-210, 600

North Wolfe Street, Baltimore, MD

21287, USA.

e-mail: akherad@jhu.edu
An intact cerebellum is a prerequisite for optimal ocular motor performance. The cerebellum fine-tunes each of the subtypes of eye movements so they work together to bring and maintain images of objects of interest on the fovea. Here we review the major aspects of the contribution of the cerebellum to ocular motor control. The approach will be based on structural-functional correlation, combining the effects of lesions and the results from physiologic studies, with the emphasis on the cerebellar regions known to be most closely related to ocular motor function: (1) the flocculus/paraflocculus for high-frequency (brief) vestibular responses, sustained pursuit eye movements, and gaze holding, (2) the nodulus/ventral uvula for low-frequency (sustained) vestibular responses, and (3) the dorsal oculomotor vermis and its target in the posterior portion of the fastigial nucleus (the fastigial oculomotor region) for saccades and pursuit initiation.

\section{Keywords: saccade, vestibular, pursuit, flocculus, paraflocculus, nodulus, vermis, fastigial}

\section{INTRODUCTION}

The cerebellum plays a pivotal role in the control of eye movements. Its core function is to optimize ocular motor performance so that images of objects of interest are promptly brought to the fovea - where visual acuity is best - and kept quietly there, so the brain has time to analyze and interpret the visual scene. The cerebellum has both immediate, on-line functions to make each individual movement accurate, and long-term, adaptive functions to keep ocular motor responses correctly calibrated to the stimuli that drive them.

\section{ANATOMICAL APPROACH TO THE CEREBELLUM AND OCULAR MOTOR CONTROL ${ }^{1}$}

The approach here will be based upon structural-functional correlations: cerebellar influences on eye movements will be discussed by anatomical regions (see Table $\mathbf{1}$ and Figure 1). Since the cerebellum has an important role in motor learning that promotes adaptation and compensation for lesions, the functional effects of focal cerebellar lesions may not strictly represent the contribution of the cerebellum to the control of eye movements in intact subjects. With this caveat, the ocular motor functions of the cerebellum will be interpreted using the combination of the effects of acute and of chronic lesions, and the results from physiological studies. Here the emphasis will be on the areas within the cerebellum about which we know most: the flocculus/paraflocculus, the nodulus/ventral uvula (lobules IX and X of the cerebellar vermis), and the dorsal oculomotor vermis (OMV; lobules V-VII) and its

\footnotetext{
${ }^{1}$ Some of this material is modified from Zee and Walker (2009).
}

target in the posterior portion of the underlying fastigial nucleus called the fastigial oculomotor region (FOR). Recent studies also implicate more lateral regions of the cerebellar hemispheres in ocular motor control.

The cerebellum has a distinctive anatomical organization. Purkinje cells of the cerebellar cortex primarily project to and inhibit cells within the underlying deep cerebellar nuclei; the vestibulocerebellum (flocculus, paraflocculus, nodulus, and uvula) also projects to the vestibular nuclei some of which can be thought of as "displaced" deep cerebellar nuclei. Axons arising from the fastigial nuclei decussate within the cerebellum and terminate primarily in the contralateral vestibular or premotor brain stem nuclei while Purkinje cells in the vestibulocerebellum project primarily to the ipsilateral brain stem nuclei.

There is a rich innervation to the cerebellar cortex from the inferior olive, through climbing fibers directly to Purkinje cells, and from premotor brain stem nuclei (as well as the vestibular nerve), through mossy fibers to cerebellar granule cells - which in turn project to Purkinje cells via parallel fibers. There are also direct climbing fiber and mossy fiber inputs to the deep cerebellar nuclei, so that the cerebellar cortex can be thought of as a parallel pathway that oversees and influences (via the projections of its Purkinje cells) the direct flow of information to and from the brain stem through the deep cerebellar nuclei.

\section{VESTIBULOCEREBELLUM: THE FLOCCULUS/PARAFLOCCULUS}

The flocculus and paraflocculus (or tonsil) together with the caudal portions of the cerebellar vermis (nodulus and uvula) are part of the oldest portion of the cerebellum or the archicerebellum, also called the vestibulocerebellum (Figure 1). Neurons within 
Table 1 | Anatomical localization of ocular motor functions within the cerebellum.

\begin{tabular}{ll}
\hline Structure & Function \\
\hline Flocculus/paraflocculus & Gaze holding \\
& Smooth pursuit and VOR cancellation \\
& Control amplitude and direction of rotational VOR \\
& Match pulse-step to prevent postsaccadic drift \\
Nodulus/ventral uvula & Downward smooth pursuit \\
& Modulate velocity-storage mechanism within vestibular nuclei to influence low-frequency \\
& rotational VOR \\
& - Amplitude and direction(relative to gravity) of rotational VOR \\
& - Habituation of rotational VOR \\
& - Tilt suppression of postrotatory VOR \\
& - Integration of otolith linear acceleration signal to velocity signals to drive translational VOR \\
Oculomotor vermis (OMV)/fastigial oculomotor & Saccade amplitude and direction \\
region (FOR) & Pursuit initiation (open-loop acceleration) \\
& Horizontal alignment
\end{tabular}

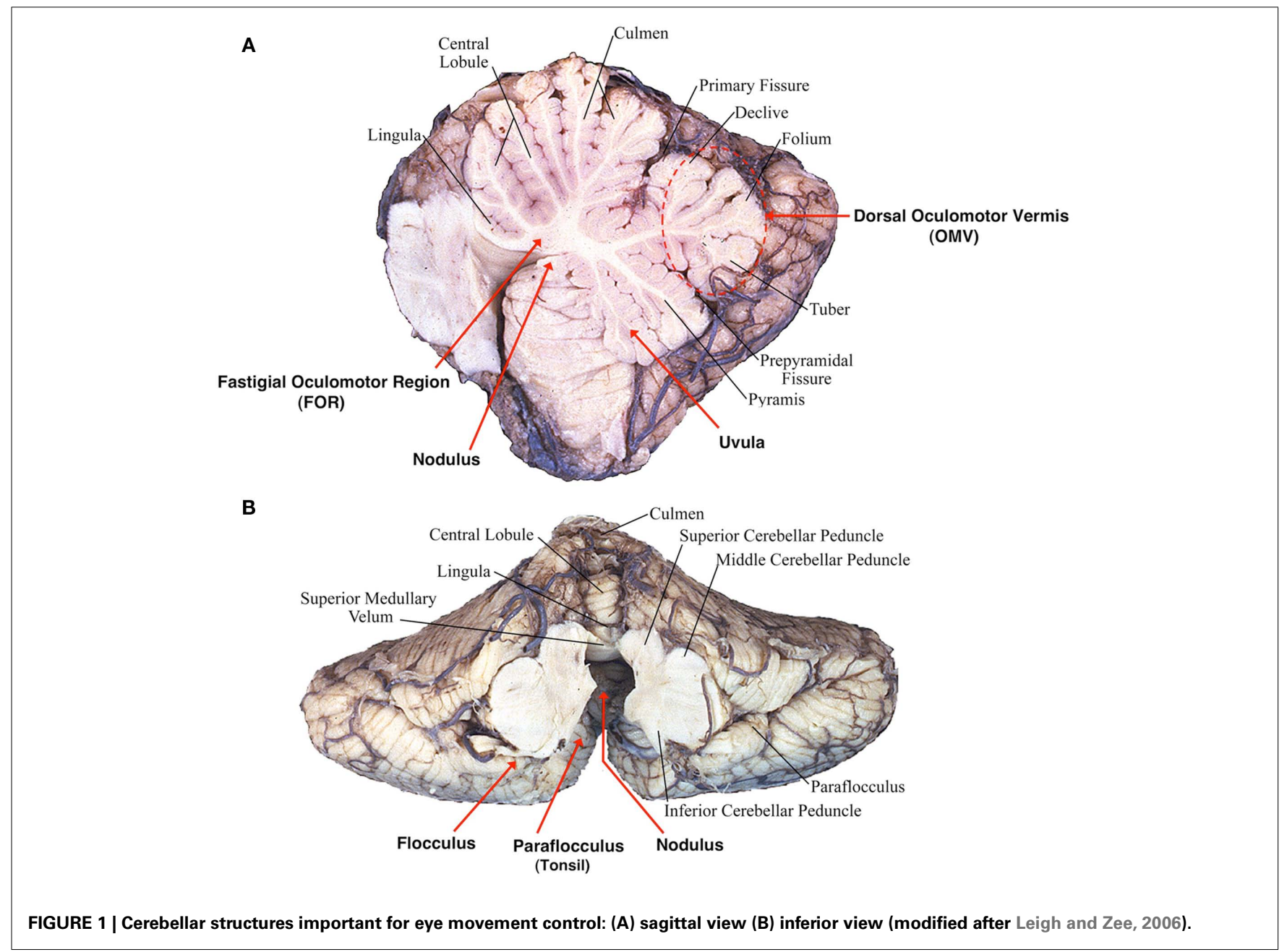

the flocculus/paraflocculus discharge in relation to the stimulus (e.g., head motion or visual target motion), to the position of the eye in the orbit during fixation, and to the movements of the eye during smooth pursuit, vergence, and vestibular responses to head motion (the vestibular-ocular reflex or VOR). Only saccades seem to be largely free of the influence of the flocculus and 
paraflocculus; they are the concern of the dorsal vermis (lobules V-VII) and its lateral extension in the cerebellar hemispheres. Note, however, that brief, several hundred milliseconds drift of the eyes after each saccade (i.e., postsaccadic drift) is a characteristic abnormality following lesions in the flocculus/paraflocculus, arising from a mismatch between the innervation that drives the eye to its new position (the "pulse") and the innervation that holds it there (the "step").

\section{PURSUIT}

Lesions of the flocculus/paraflocculus impair smooth tracking of a moving target, either when the head is still (smooth pursuit) or passively moving (VOR cancellation; Zee et al., 1981; Belton and McCrea, 2000, 2002; Rambold et al., 2002). Both the initiation of pursuit and the response during sustained tracking can be affected. The floccular complex seems to play a lesser role with active head turns than passive head rotations during smooth tracking (Belton and McCrea, 1999, 2004).

Complete lesions of the flocculus/paraflocculus lead to a decrease in gain (eye velocity/target velocity) of pursuit during steady-state (constant-velocity) tracking. Even with relatively complete floccular/parafloccular lesions pursuit function recovers considerably. This is likely mediated by other parts of the cerebellum as studies in monkeys have shown that Purkinje cells in the dorsal vermis and its lateral extension in the cerebellar hemisphere are also involved in the control of smooth pursuit, and they share some mossy and climbing fiber inputs with the paraflocculus (Ohki et al., 2009; Xiong et al., 2010). In humans, too, smooth pursuit is affected by transcranial magnetic stimulation (TMS) of the skull over the dorsal vermis (Hashimoto and Ohtsuka, 1995; Ohtsuka and Enoki, 1998). More caudal regions of the vermis (i.e., the uvula and nodulus) are also implicated in the control of smooth tracking (Heinen and Keller, 1996; Walker et al., 2008b).

An inherent functional asymmetry for vertical pursuit eye movements has been described within the flocculus. The majority of Purkinje cells of the flocculus are active during downward as opposed to upward pursuit (Stone and Lisberger, 1990). This "updown" asymmetry during vertical pursuit is also apparent during functional imaging of the flocculus in healthy human subjects (Glasauer et al., 2009).

\section{GAZE HOLDING}

A second cardinal feature of lesions of the flocculus/paraflocculus is impaired gaze holding with the eyes drifting centripetally after eccentric eye movements, resulting in a gaze-evoked nystagmus (Zee et al., 1981; Figure 2). Thus the flocculus/paraflocculus functions in the control of the brain stem circuits that convert (mathematically integrate) velocity into position commands for all types of conjugate eye movements, and so assure steady fixation.

A simple hypothesis to explain the effects of lesions of the flocculus/paraflocculus on gaze holding is based upon the idea that there is an inherently poor, "leaky" ocular motor integrator within the brain stem. The neural integrator sustains the discharge of ocular motor neurons to generate a tonic eye position command in the absence of any ongoing external input (e.g., a saccade command; Figure 2). The neurons in the paramedian tracts and in the medial vestibular nucleus (MVN) and the nucleus prepositus hypoglossi are likely important constituents of this integrator network, and the superior vestibular nucleus and the interstitial nucleus of Cajal in the midbrain may play a similar role in the neural integration for vertical and torsional eye position commands. In this scenario, the flocculus/paraflocculus modulates activity in a positive feedback

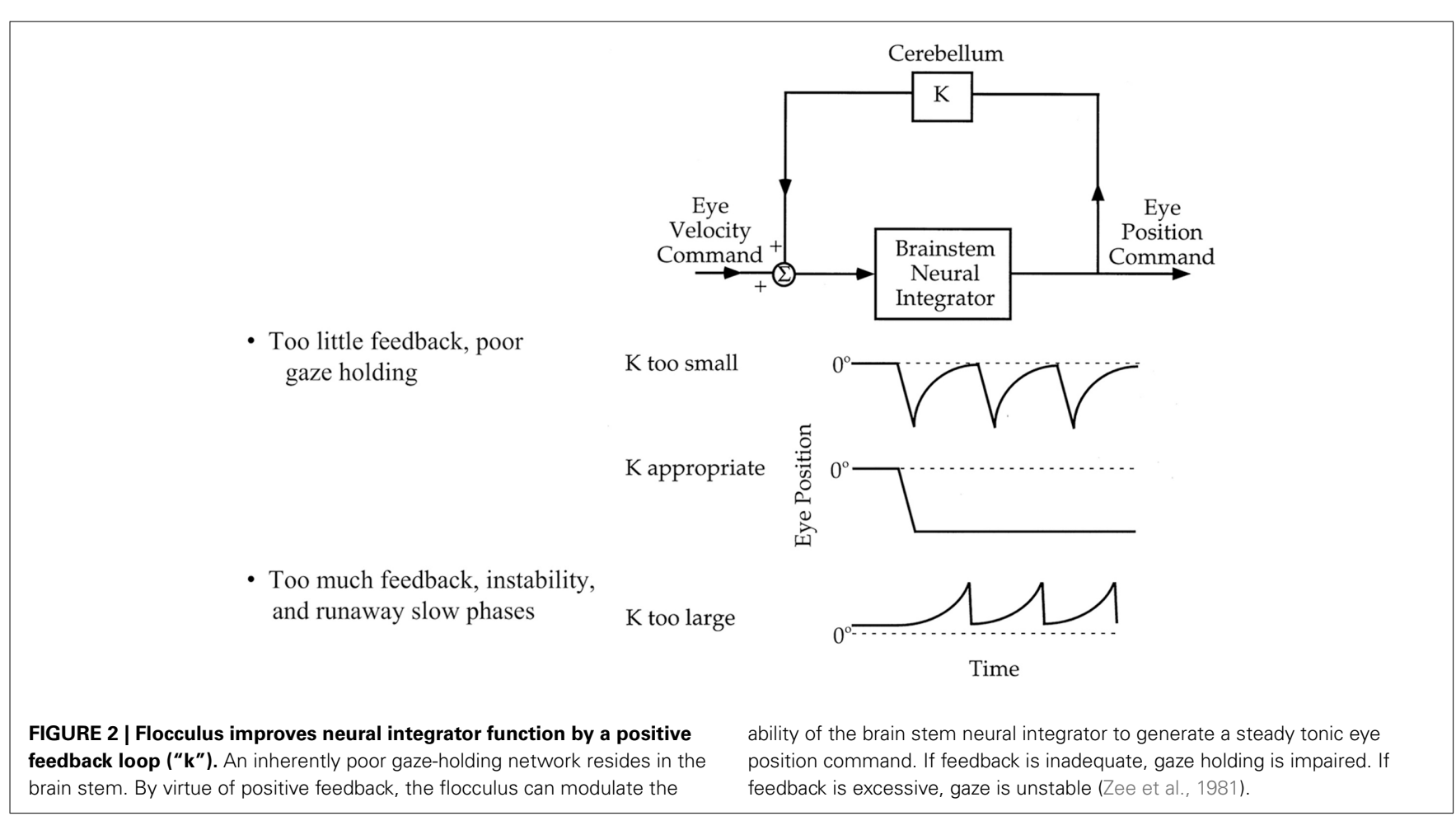


loop around the brain stem neural integrator and improves its performance.

\section{SPONTANEOUS NYSTAGMUS}

A third distinctive feature of lesions in the flocculus/paraflocculus is downbeat nystagmus, in which the eyes drift up (slow phase) and are brought back to the fixation target by a corrective downward saccade (quick phase). This form of nystagmus can be linked to the damage of the physiologic "up-down" asymmetry of floccular Purkinje cells (with predominant downward facilitation) resulting in upward slow drift (Marti et al., 2005, 2008; Kalla et al., 2006; Hufner et al., 2007). An alternative hypothesis is based on the tonic inhibition by the flocculus upon the upward VOR (by inhibitory projections to the superior vestibular nucleus), and lack of corresponding projections from the flocculus to the brain stem structures that mediate downward vestibuloocular responses (reviewed in Leigh et al., 2002). According to this hypothesis, damage to the flocculus could release the tonic inhibition upon the vestibular nuclei associated with the upward VOR and therefore lead to slow, upward drifts of the eyes followed by corrective, quick phases downwards (downbeat nystagmus).

A curious feature of the upward drift is the variability of its waveform from subject to subject. For example, monkeys with experimental flocculus/parafloccular lesions show downbeat nystagmus with either a decreasing- or an increasing-velocity waveform (Zee et al., 1981). These different velocity profiles suggest differences in the effect of the lesion on the vertical neural integrator. If the integrator is sufficiently "leaky" (in the absence of a new external input its output decays rapidly with time), the velocity of the slow phase will be decreasing. If the integrator is "unstable" (in the absence of a new external input its output increases with time), the velocity of the slow phase will be increasing. For the horizontal system, monkeys with floccular/parafloccular lesions always develop a gaze-evoked nystagmus with a velocity-decaying waveform, indicating that the flocculus/paraflocculus is critical for improving the performance of the inherently "leaky" horizontal brain stem integrator (Zee et al., 1981). The variable waveform of the upward slow phases of the downbeat nystagmus associated with lesions of the flocculus/paraflocculus suggests that for the vertical integrator the flocculus/paraflocculus has a more subtle, modulatory role, possibly related to the long-term adaptation capability of an individual animal. In other words, based upon the animal's own ocular motor history (e.g., trauma or disease) and genetic makeup, the inherent brain stem vertical neural integrator could be relatively leaky or relatively unstable, and the cerebellar lesion then unmasks the "default" behavior (e.g., Zee et al., 1981).

Cerebellar patients with downbeat nystagmus may also show velocity-increasing wave forms. In these patients the nystagmus becomes more intense when the patient changes eye position to look in the direction of the slow phase, the opposite of the common pattern in which the nystagmus becomes more intense when the patient looks in the direction of the quick phase (Alexander's law). 3,4-Diaminopyridine (3,4-DAP) and 4-Aminopyidine (4-AP), potassium channel blockers, can diminish downbeat nystagmus associated with cerebellar lesions (Strupp et al., 2003; Helmchen et al., 2007; Kalla et al., 2007). This may be by restoring the precision of pacemaking in Purkinje cells through blocking potassium channels and prolonging the action potential in these cells (Alvina and Khodakhah, 2010).

Another form of nystagmus typically seen in patients with cerebellar syndromes is rebound nystagmus (Lin and Young, 1999; Hashimoto et al., 2003). The nystagmus is short-lived and occurs when the eyes are returned to the central position following sustained eccentric gaze. The rebound nystagmus beats oppositely to the prior gaze-evoked nystagmus, i.e., the slow phase is toward the prior eccentric gaze position. Similar to gaze-evoked nystagmus, rebound nystagmus is linked to the gaze-holding neural integrator controlled by the vestibulocerebellum. Rebound nystagmus is also present in normal subjects if fixation is removed when the eyes are returned to the straight-ahead position after sustained eccentric gaze. This phenomenon presumably reflects the action of a normal adaptive mechanism (which is also intact or even becomes excessive in cerebellar syndromes) that changes the set point or null position of the gaze-holding network toward the eye position that is most frequently used during fixation. In some patients the mechanism producing rebound nystagmus becomes unstable leading to a centripetal-beating nystagmus on eccentric gaze in which case slow phases are directed outwards.

\section{POSTSACCADIC DRIFT}

A brief drift of the eyes lasting several hundred milliseconds following each saccade, is another feature of the floccular/parafloccular syndrome. The postsaccadic drift reflects a mismatch between the pulse (phasic) and the step (tonic) components of innervation that drives saccades and is due to the abnormal amplitude of the step output of the neural integrator relative to its velocity input, i.e., the pulse. Normally these two premotor signals are matched precisely, so the eye abruptly stops at the end of the saccade. In monkeys with lesions of the flocculus/paraflocculus, the direction of the postsaccadic drift, onward, or backward, is variable from animal to animal, again in keeping with a modulatory role of the cerebellum upon the inherent capabilities of the premotor brain stem circuits generating eye movements (Zee et al., 1981).

\section{VESTIBULAR-OCULAR REFLEXES}

The flocculus and paraflocculus are not critical for generating a compensatory response to head rotations per se, in the sense that the VOR is still present in animals with ablation of the flocculus/paraflocculus or even after complete ablation of the cerebellum. Nevertheless, different regions of the vestibulocerebellum modulate the VOR. Visual inputs are crucial for a long-term adaptive mechanism that allows the brain to monitor the VOR and correct its performance. This adaptive capability can change the gain, the direction, or the phase (temporal relationship between input and output) of the VOR, each of which reduces image motion during head turns (Figure 3). Bilateral ablation of the flocculus and ventral paraflocculus results in an inability of an animal to undergo adaptive VOR changes and has a variable but persistent effect on the amplitude of the VOR gain (Takemori and Cohen, 1974; Zee et al., 1981; Lisberger et al., 1984; Rambold et al., 2002).

During rotation of the head around an earth-vertical axis, patients with diffuse cerebellar lesions may show a dynamic 
upward bias so that the eyes move up as well as horizontally, producing a "cross-coupled" VOR (Walker and Zee, 1999, 2005a; Shaikh et al., 2011; Figure 4). There are also inappropriate torsional components and the responses in the two eyes are disconjugate, with relatively more torsion in one eye and more vertical rotation in the other. A release of inhibition upon anterior semicircular canal pathways within the brain stem (which produce upward slow phases) is a plausible explanation (Zhang et al., 1995; Walker and Zee, 2005a). In line with this hypothesis, patients with

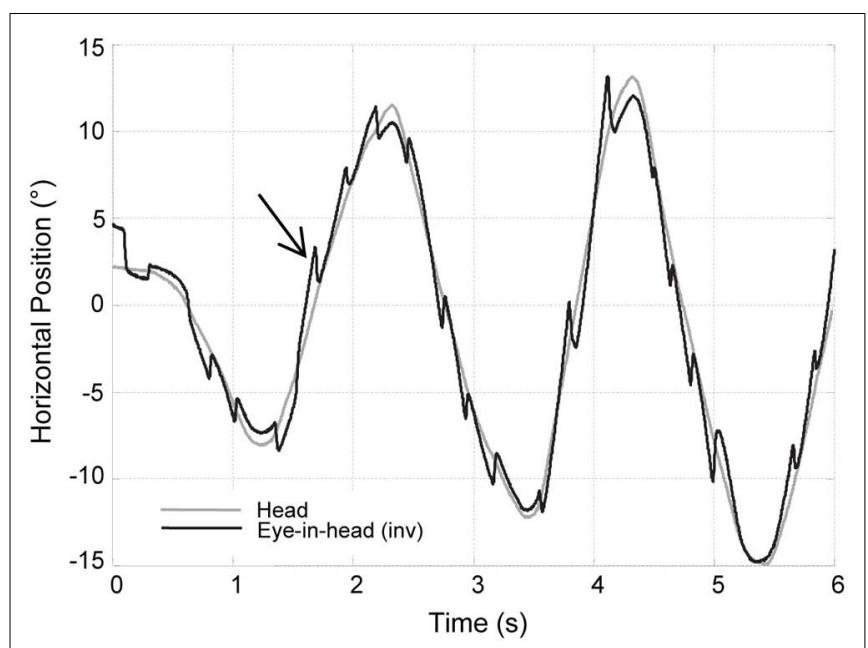

FIGURE 3 | Increased VOR gain with cerebellar disease. The inverse of horizontal eye position (black trace) is superimposed on the head position (gray trace) while the patient is fixating on a target during sinusoidal head rotation. Frequent corrective, back up saccades (black arrow) are required to stay on the target and compensate for increased VOR gain. cerebellar disease have an asymmetric vertical VOR with higher gain for downward head impulses (consistent with increased anterior semicircular canal stimulation; Walker and Zee, 2005b; Shaikh et al., 2011). These results implicate the cerebellum, and likely the flocculus/paraflocculus, in generating movements of each eye that have the correct amplitude and direction for perfect VOR compensation.

The cerebellum also plays a role in the translational VOR $(\mathrm{t}$ VOR). The t-VOR can be measured alone (e.g., pure side-to-side, fore-aft, or up-down translation), or with a rotation of the head when the axis is in front of or behind the labyrinths which leads to a combined rotation and translation. In patients with cerebellar disease the t-VOR can be profoundly impaired even when the rotational VOR (r-VOR) is intact (Baloh et al., 1995; Zee et al., 2002). These patients also have defects in adjusting the gain of the r-VOR for the distance of the target of interest, and for the displacement of the orbits away from the axis of head rotation. These are necessary requirements for adequate gaze stabilization during head rotation, since virtually all head rotations are accompanied by some translation of the head and specifically of the orbits. The flocculus/paraflocculus has neurons that discharge in relation to vergence and it is possible that this information is used to adjust the VOR for target distance (Miles et al., 1980). Other parts of the cerebellum (e.g., nodulus, ventral uvula) also play a role in the t-VOR (Shaikh et al., 2005; Walker et al., 2008a, 2010).

\section{VESTIBULOCEREBELLUM: THE NODULUS/VENTRAL UVULA}

The nodulus and adjacent ventral uvula are the most caudal aspects of the cerebellar vermis (Figure 1). They mainly contribute to the control of the rotational and the t-VOR. Lesions in the nodulus/uvula also alter smooth pursuit and optokinetic nystagmus

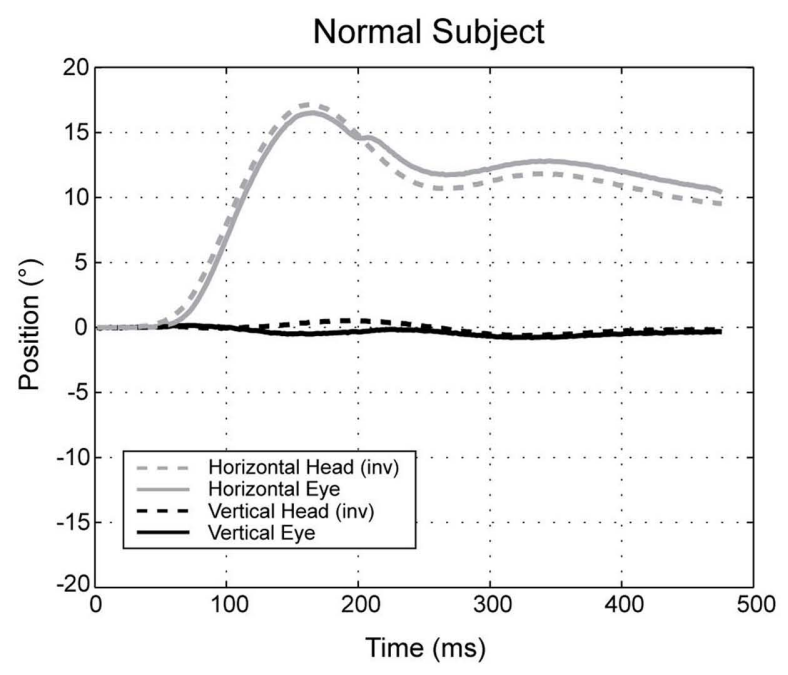

FIGURE 4 | Position plots of representative yaw impulse responses in a normal subject and a patient with cerebellar disease. Horizontal and vertical head (inverted) and eye-in-head positions are shown for rightward yaw impulses, starting from the center position. For the patient, the change in horizontal eye position exceeds the change in head position (i.e., the gain is high). At the same time, the eye moves up in the orbit, even though there is

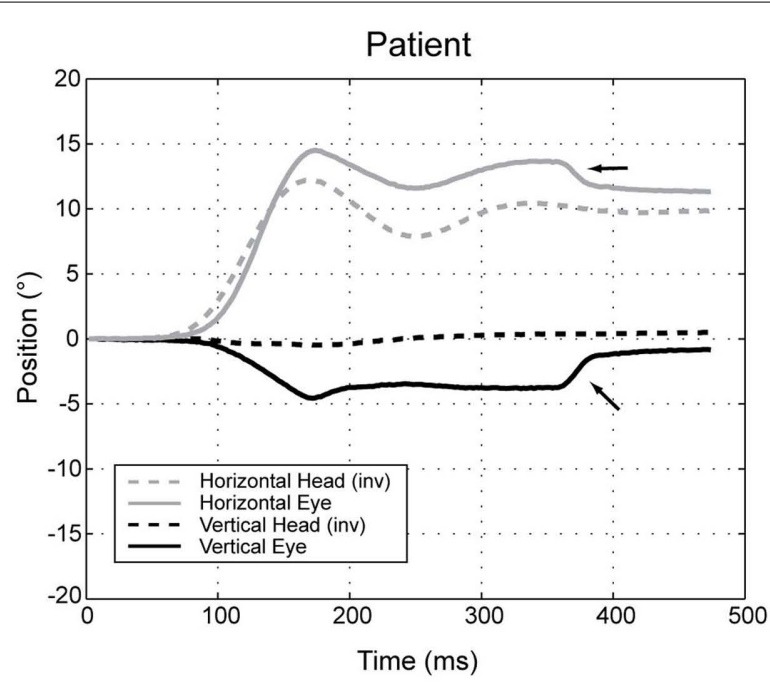

essentially no downward head movement. The arrows indicate horizontal and vertical components of a saccade that partially corrects the gaze position error. Right-hand-rule conventions are used: positive positions are leftward and downward, except for head positions, which are inverted to facilitate comparison (reprinted from Walker and Zee (2005a), with permission from the American Physiologic Society). 
(OKN), but their exact contribution to these visual-following reflexes is unclear.

\section{VESTIBULAR-OCULAR REFLEXES}

The nodulus/ventral uvula act upon the "low-frequency" (sustained) components of the VOR via projections to a "velocitystorage" mechanism within the vestibular nuclei. With a constantvelocity rotation of the head, the velocity-storage mechanism extends the duration of the VOR response beyond that expected from the mechanical properties of the cupula-endolymph system within the semicircular canals. This perseverating (integrating) action slows the decay of nystagmus that normally occurs during a constant-velocity rotation in the dark. In other words, velocitystorage improves the performance of the horizontal VOR at low frequencies of rotation and so extends the "bandwidth" of frequencies over which the horizontal VOR can transduce the motion of the head into the correct compensatory eye movement. Secondly, during sustained "off-vertical axis" rotation of the head, when there is an imposed changing linear acceleration due to the continuous reorientation of the head relative to the pull of gravity, the velocitystorage mechanism modulates the direction of compensatory slow phases, reorienting the axis of eye rotation toward earth-vertical. It thus serves an orienting function so the brain can know the position of the head relative to the pull of gravity, as well as determine whether a sensed linear acceleration of the head is from gravity or an imposed translation of the head (see below for a discussion of the tilt-translation ambiguity).

Lesions of the nodulus/uvula alter the velocity-storage mechanism for the horizontal VOR and increase the duration of vestibular responses to a constant-velocity input around an earth-vertical axis (i.e., the VOR time constant is increased; Waespe et al., 1985). The effect of nodulus/uvula lesions on the vertical and torsional VOR, however, is the opposite; their time constants are shortened (Waespe et al., 1985; Angelaki and Hess, 1994; Wearne et al., 1998). Lesions of the nodulus/uvula also disrupt the spatial orientation function of the velocity-storage mechanism; the VOR no longer reorients the axis of eye rotation toward upright during offvertical axis rotation (Wearne et al., 1998; Cohen et al., 2002). With nodulus/uvula lesions, there is also a loss of the normal habituation of the time constant of the VOR to repetitive stimulation as well as loss of tilt suppression of postrotary nystagmus, the phenomenon by which the decay of postrotary nystagmus is hastened with pitching the head down immediately following the end of a constant-velocity rotation (Hain et al., 1988; Cohen et al., 1992).

As mentioned earlier, patients with diffuse cerebellar disease may show abnormally directed slow phases, or "cross-coupling" of their VOR during high-frequency, high acceleration impulse testing (Figure 4). This phenomenon also occurs during lowfrequency head rotation around an earth-vertical axis, during sustained optokinetic stimulation with the head upright, or after horizontal head shaking (Walker and Zee, 1999; Kim et al., 2005; Moon et al., 2009). The "cross-coupling" with low-frequency stimuli likely reflects dysfunction of the nodulus/uvula and the inability to control spatial orientation using the velocity-storage mechanism.

The nodulus/uvula also plays a role in other aspects of otolithcanal interaction including solving the tilt-translation ambiguity.
The otoliths signal linear acceleration regardless of its source, and cannot in themselves distinguish between a dynamic translation of the head and a static reorientation of the head with respect to gravity (Yakusheva et al., 2008). It is the simultaneous presence or absence of signals from the vertical semicircular canals that resolves the issue of tilt in the roll plane (ear to shoulder) versus translation.

The nodulus/uvula is also important for the processing of otolith signals from both the utricle and saccule to drive the t-VOR response. They are involved in the integration of linear head acceleration signals to velocity signals derived from otolith afferents (Walker et al., 2010). Ablation of the nodulus/uvula in monkeys impairs the ocular response to vertical translation and reduces eye velocity during sustained horizontal translation along the interaural direction (Walker et al., 2008a). The reduction in the interaural $\mathrm{t}$-VOR occurs only in the dark, whereas the vertical t-VOR is substantially impaired even in the light. These finding suggest the nodulus/uvula might have different contribution to the horizontal and vertical t-VOR.

\section{PURSUIT}

Although the flocculus/paraflocculus is primarily implicated in the control of pursuit, recent evidence also indicates a role for the nodulus/uvula, particularly in vertical pursuit, as experimental lesions of the nodulus/uvula impair downward pursuit with little effect on upward or horizontal pursuit (Walker et al., 2008b). A similar finding of impaired downward pursuit has been reported in a patient with an isolated nodulus/uvula lesion (Helmchen et al., 2007). The uvula has been linked to horizontal smooth pursuit by lesion mapping in a group of patients with cerebellar stroke (Baier et al., 2009). In this study, however, the identified lesions were not isolated to the uvula and involved other parts of the vermis.

\section{OPTOKINETIC REFLEX}

The optokinetic response is normally judged by both the nystagmus during visual stimulation $(\mathrm{OKN})$ and the slowly decaying after-nystagmus (OKAN) in the dark that occurs after the stimulation has ceased. OKAN shares the same velocity-storage mechanism with the VOR. The smooth pursuit system also participates at the onset of the response to an optokinetic (full-field) stimulus bringing the eyes quickly to the maximum velocity. Accordingly, the cerebellar regions involved in generating both pursuit and the VOR contribute to the tracking response to an optokinetic stimulus. For example, after flocculectomy in monkeys, due to the coexisting pursuit deficit the initial eye velocity during constantvelocity optokinetic stimulation is diminished, and the time to increase velocity from the onset of the optokinetic stimulus to a steady-state (matching the drum velocity) is prolonged (Zee et al., 1981). After removal of the nodulus/uvula, analogous to the VOR responses, horizontal OKAN is prolonged but the vertical and torsional OKAN response times are shortened (Angelaki and Hess, 1994; Wearne et al., 1998). Similarly, spatial control of the eye velocity vector during OKN or OKAN is impaired and the axis of eye rotation during imposed gravito-inertial acceleration does not reorient toward the upright (Cohen et al., 2002). There is also a change in the direction of the optokinetic response that results 
in cross-coupling during OKN or OKAN (a dynamic torsional or upward bias with horizontal nystagmus; Walker and Zee, 1999).

\section{SPONTANEOUS NYSTAGMUS}

Periodic alternating nystagmus (PAN), a horizontal jerk nystagmus that changes direction every few minutes, may appear following lesions of the nodulus and its adjacent paravermal region (Waespe et al., 1985). PAN reflects the combined actions of a (1) disinhibited brain stem vestibular velocity-storage mechanism (due to loss of inhibition from Purkinje cells in the nodulus that project to the vestibular nuclei) and (2) an intact adaptive mechanism that acts to null any sustained unidirectional nystagmus, thus allowing PAN to change direction (Leigh et al., 1981). Because Purkinje cell inhibition is mediated through $\mathrm{GABA}_{\mathrm{B}}$ receptors, treatment with baclofen (a GABA $\mathrm{B}$ agonist) disengages the velocity-storage mechanism and stops PAN (Halmagyi et al., 1980; Cohen et al., 1987). Note that like the mechanism producing rebound nystagmus, the adaptive mechanism that leads to the reverse of the direction of PAN is intact or possibly increased after lesions in the cerebellum.

Downbeat nystagmus is also reported with nodulus and uvula lesions (Walker et al., 2008b, 2009). The slow phase velocity of this nystagmus, unlike down beat nystagmus seen with flocculus lesions, is independent of orbital position (i.e., nystagmus did not decrease with upward gaze or increase with lateral gaze) and can be suppressed with visual fixation. Changing the orientation of the head with respect to gravity may also alter the nystagmus (Walker et al., 2009). Thus the downbeat nystagmus with nodulus/uvula lesions could be due to a bias in the vestibular system (either the $\mathrm{t}$-VOR or r-VOR mechanisms) and need not reflect changes in the gaze-holding neural integrator.

\section{FUNCTIONAL DIFFERENCES BETWEEN THE FLOCCULUS/PARAFLOCCULUS AND THE NODULUS/UVULA}

The vestibulocerebellum has many functions and lesions produce a variety of abnormalities. Are there overriding principles about the functions of the two areas within the vestibulocerebellum? One simple hypothesis is that the flocculus and paraflocculus are more concerned with the relatively immediate and fast-acting aspects of ocular motor function that relate to the needs of the fovea (e.g., holding images steady on the fovea during attempted fixation, smooth pursuit, VOR, or immediately following saccades). On the other hand, the nodulus and ventral uvula are more concerned with the duration and axis of eye rotation in response to low-frequency, sustained rotational stimuli, and so determine the orientation of images on the retina relative to upright. Recent evidence however indicates that an intact nodulus and uvula are also necessary for the proper function of the high-frequency t-VOR as well as smooth pursuit (Walker et al., 2008b, 2010). Thus there does not appear to be an absolute compartmentalization of functions of the VOR and pursuit among the different parts of the vestibulocerebellum, and, as will be discussed below, the dorsal vermis and underlying fastigial nuclei also play a role in smooth pursuit. Such redundancy is certainly an advantage during locomotion, considering the vital role vestibular and pursuit responses play in stabilizing gaze on stationary targets or in following targets that are also moving in the environment.

\section{DORSAL CEREBELLAR VERMIS AND THE POSTERIOR FASTIGIAL NUCLEUS}

The dorsal vermis (lobules V-VII, also called the OMV) and the underlying posterior fastigial nucleus (also called the FOR) are especially important for the control of saccades, but also play a role in smooth pursuit (Figure 1).

\section{SACCADES}

Lesions in the OMV cause changes in the accuracy, latency, trajectory, and dynamic properties (speed and acceleration) of saccades (Takagi et al., 1998; Barash et al., 1999; Kojima et al., 2010a). Purkinje cells in the OMV discharge before saccades, and stimulation of this same area can elicit saccades (Fujikado and Noda, 1987; Noda and Fujikado, 1987). In humans, too, artificial stimulation using TMS, functional magnetic resonance imaging (fMRI), and mapping of lesions based on MRI in patients with cerebellar infarctions confirm the participation of the OMV in the generation of saccades (Hashimoto and Ohtsuka, 1995; Hayakawa et al., 2002; Ye et al., 2010). The OMV also plays an important role in saccade adaptation, a mechanism that detects errors in motor performance and updates saccade commands to accurately move the eye toward a target. OMV lesions impair adaptation of saccade amplitude (Takagi et al., 1998; Barash et al., 1999; Straube et al., 2001; Jenkinson and Miall, 2010; Colnaghi et al., 2011). This defect in part may be related to the increased variability of saccade amplitude after the lesion of the OMV, so that the adaptation mechanism no longer receives a consistent error signal with which it can optimally reprogram the correct response.

Neurons in the FOR also discharge in relation to saccades (Ohtsuka and Noda, 1991; Fuchs et al., 1993). They supply a presaccadic burst for contraversive saccades (i.e., the right FOR bursts before leftward saccades) and a "braking" discharge, late during the saccade, for ipsiversive saccades (the right FOR bursts toward the end of rightward saccades). Thus, each FOR acts to facilitate contraversive saccades and contributes to the termination of ipsiversive saccades. Consequently, lesions in the FOR cause ipsiversive saccadic hypermetria (overshoot) and contraversive hypometria (undershoot; Robinson et al., 1993). A horizontal static fixation error (toward the side of the lesion) could also contribute to the saccadic dysmetria observed following FOR lesions (Robinson et al., 1993). Bilateral FOR lesions cause bilateral hypermetria.

The OMV receives information about performance during saccades and adjusts its inhibition upon the FOR to assure the saccade arrives on target (Goffart et al., 2003; Straube et al., 2009). Purkinje cells in the OMV behave in a similar way to those of the FOR, though, as predicted from their inhibitory nature, their "sign" is opposite. Thus, each side of the vermis acts to facilitate ipsiversive saccades and contributes to the termination of contralateral saccades. Accordingly, OMV lesions lead to hypometric ipsiversive and hypermetric contraversive saccades (Takagi et al., 1998). Bilateral lesions in OMV cause hypometric saccades in both horizontal directions.

The role of the cerebellum in the generation of vertical saccades is not as well understood. Vertical saccades show ipsipulsion (oblique trajectory toward the side of inactivation) with experimental lesions of the FOR (Straube et al., 2009). Ipsipulsion is also a feature of Wallenberg's syndrome, presumably due to a 
functional lesion of the FOR resulting from interruption of the climbing fiber input (within the inferior cerebellar peduncle) to the OMV and a consequent increased inhibition by Purkinje cells upon the underlying FOR (Waespe and Wichmann, 1990). Other areas, such as the interposed nucleus (IP; emboliform and globose) and paraflocculus (tonsils), may also be important in the generation of vertical saccades (Robinson, 2000).

The central role of the cerebellum in the control of saccades is reflected in the different ways it can influence the trajectory of the saccade. First, it provides an additional drive that increases the acceleration of the eyes to its maximum velocity; second, it monitors the progress of the saccade and adjusts the trajectory as needed to keep the eye on track to arrive on target; and third, it helps to end the saccade at just the right time by choking off the saccadic pulse drive. But how does the cerebellum influence the brain stem circuits that generate saccades? There are many targets by which the cerebellar output, via FOR, could influence saccades (Figure 5; Noda et al., 1990; Quaia et al., 1999). The early influence of the FOR on the initiation of saccades could be mediated through the excitatory burst neurons (EBN) within the brain stem reticular formation. The late influence of the FOR on the ending

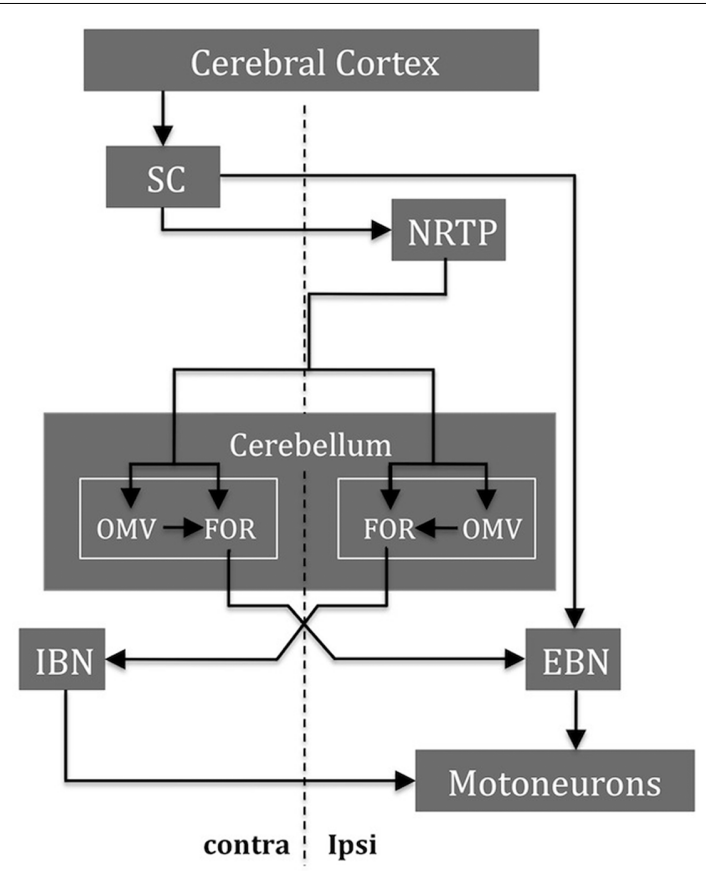

FIGURE 5 | Some major structures and their main connections are shown for saccade control in one direction. During the saccade, the cerebellum integrates the efference copy of the drive signal from the NRTP (ipsilateral to the movement) that projects bilaterally to both OMV and FOR within the cerebellum. The SC and the FOR (both contralateral to the movement) excite the EBN that contact the motoneurons (both ipsilateral to the movement) of the agonist muscle. When the eyes approach the target, the ipsilateral FOR neurons produce a choke signal through the contralateral IBN that inhibits the motoneurons of the agonist muscle. NRTP, nucleus reticularis tegmenti pontis; DLPN, dorsolateral pontine nuclei; OMV, oculomotor vermis; FOR, fastigial oculomotor region; SC, superior colliculus; EBN, excitatory burst neurons; IBN, inhibitory burst neurons (modified after Quaia et al., 1999). of saccades could be through the inhibitory burst neurons (IBN) also in the brain stem reticular formation. These neurons could act to "choke" or "brake" the saccade by strongly inhibiting the abducens nucleus that had been driving the agonist muscles during the saccade. The activity of the FOR during saccades is further modulated by the Purkinje cells of the overlying dorsal vermis (reviewed in Gad and Anastasio, 2010). Projections of the FOR to omnipause neurons (OPN), which inhibit premotor saccadic EBN - either directly or via projections to OPN from the fixation zone of the rostral pole of the superior colliculus - are other routes by which the FOR could help bring the saccade to an end.

Lesions in different parts of this circuitry could produce different patterns of abnormalities of saccades. For example, disinhibition of the FOR might lead to saccadic oscillations (back to back saccades without an intersaccadic interval) by increasing the discharge rate of both EBN and IBN neurons. This has been suggested as a cause of opsoclonus (e.g., in paraneoplastic and other immune mediated syndromes; Wong et al., 2001; Helmchen et al., 2003). Dysfunction of the FOR might also lead to saccadic intrusions, as occur in spinocerebellar ataxia syndromes (Pula et al., 2010). Saccadic intrusions and hypermetric saccades occur in SCASI (spinocerebellar ataxia with saccadic intrusions) in which patients also have peripheral neuropathy. It has been suggested that slowed nerve conductions along parallel fibers within the cerebellar cortex could lead to a delay in the feedback control of saccade termination by the cerebellum and in this way cause hypermetric saccades (Swartz et al., 2003). Memantine, a non-competitive blocker of the NMDA receptor, can reduce saccadic intrusions in some of these patients likely by its action on the deep cerebellar nuclei (Serra et al., 2008). Spinocerebellar ataxia type 2 (SCA2) is associated with slowing of saccades without loss of accuracy and presumably relates to a loss of burst neurons in the brain stem reticular formation. The finding of slow saccades in otherwise asymptomatic carriers of the SCA2 mutation could be due to degeneration of the burst neurons within the pons earlier than degeneration in the cerebellum itself (Velazquez-Perez et al., 2009). In contrast to SCA2 patients, in patients with SCA6, and those with a similar phenotype but without the SCA6 genetic mutation (so-called late onset cerebellar ataxia; LOCA) the velocity of saccades is relatively preserved but the accuracy is impaired (Federighi et al., 2011). Presumably, degeneration in the OMV/FOR causes the saccadic dysmetria in patients with LOCA and SCA6.

\section{PURSUIT}

The OMV and FOR also participate in the generation of pursuit eye movements. Electrical stimulation of the OMV in monkeys can enhance contraversive or impair ipsiversive pursuit, and TMS of the skull over the posterior cerebellum in humans can influence pursuit eye movements in the same pattern (Krauzlis and Miles, 1998; Ohtsuka and Enoki, 1998). There are neurons in both the OMV and the FOR that discharge in relation to smooth pursuit (Suzuki and Keller, 1988; Fuchs et al., 1994). In the case of the FOR, neurons discharge early during contraversive pursuit and late for ipsilateral pursuit, analogous to activity associated with saccades. Thus, each FOR can facilitate contraversive pursuit and can contribute to the termination of ipsiversive pursuit. Purkinje cells in the OMV probably behave in a similar way to those of the FOR, 
though, as predicted from their inhibitory nature their "sign" is opposite. Each side of the vermis would act to facilitate ipsiversive pursuit and contribute to the termination of contralateral pursuit. Purkinje cells in the OMV encode the sum of eye velocity in the orbit and retinal slip velocity (the rate of image motion across the retina) during smooth pursuit that together represent target velocity relative to the head (Suzuki et al., 1981). These Purkinje neurons in the vermis differ from those in the flocculus/paraflocculus that modulate their discharge with eye velocity but not with the retinal slip velocity (Belton and McCrea, 2000).

The pursuit deficits reported after experimental lesions in the OMV and the FOR are largely in accord with the physiological findings (Robinson et al., 1997; Takagi et al., 2000). With a lesion in the FOR contralateral pursuit is impaired, and with a lesion in the OMV ipsilateral pursuit is impaired. Vertical pursuit is little affected following OMV lesions whereas FOR lesions reduce downward pursuit more than upward pursuit. Bilateral lesions of the OMV in monkeys produce horizontal pursuit deficits in both directions (Takagi et al., 2000). On the other hand, bilateral FOR lesions leave pursuit relatively intact (Robinson et al., 1997). This also appears to be the case in patients with bilateral FOR lesions in whom pursuit is preserved (Buttner et al., 1994). These finding suggest the pursuit deficit is due to imbalance between opposing drives of the two FOR. Therefore, with bilateral FOR inactivation, and no FOR imbalance, the pursuit movements remain intact.

Lesions of OMV and FOR mainly affect eye acceleration during the initial period of pursuit (the first $100 \mathrm{~ms}$ of tracking after a target has started moving or has changed its speed) and have a smaller effect during the sustained tracking period (Robinson et al., 1997; Takagi et al., 2000). During this initial “open-loop" period, an eye acceleration command brings the eye toward a certain velocity prior to the sustained tracking period. The inherent latency in visual processing requires the pursuit system to be able to calibrate the initial response adaptively, as opposed to the period of sustained tracking in which "on-line" visual feedback and predictive mechanisms can be used to keep pursuit accurate (Kettner et al., 2002). Lesions of the OMV also interfere with pursuit adaptation (Takagi et al., 2000).

As mentioned earlier, in addition to OMV/FOR, the flocculus/paraflocculus of the vestibulocerebellum has neurons that discharge with pursuit eye movements. Thus, both the vestibulocerebellum and the OMV/FOR contribute to smooth pursuit. One possible division of labor between these two regions is that the OMV/FOR is more concerned with the initiation and termination of the preprogrammed initial "open-loop" portion of pursuit (when retinal slip is high), and the vestibulocerebellum is more concerned with pursuit during sustained tracking.

A remarkable finding after OMV lesions in monkeys is that there are quantitative similarities in the effects of the lesions on saccade amplitude, and on pursuit velocity during the "open-loop" initial period of pursuit tracking (Takagi et al., 1998, 2000). This similarity is seen more clearly if one considers that the initial period of pursuit acceleration is an open-loop eye movement in the same vein as a saccade is open-loop. With this hypothesis, for saccades, premotor networks would be generating an eye velocity command to bring the eye to a certain position, and for pursuit, an eye acceleration command to bring the eye toward a certain velocity. Taking this analogy further, the FOR and the OMV could control the dynamic properties of the open-loop period of pursuit (as reflected in eye acceleration) in the same way that they control the dynamic properties of saccades (as reflected in eye velocity). Compatible with this idea is the finding of neurons within the OMV that discharge for both pursuit and saccades, and that stimulation of the OMV can alter ongoing saccades or pursuit, depending upon the parameters of the stimulus (Sato and Noda, 1992).

Figure 6 shows a simplified diagram of the major cerebellar and brain stem structures involved in generating horizontal pursuit. The pontocerebellar projections, in particular from the nucleus reticularis tegmenti pontis (NRTP) and the dorsolateral pontine nuclei (DLPN), are major links for transmission of pursuit signals from higher cortical regions to the cerebellum. The brain stem afferents from the DLPN project more strongly to the flocculus/paraflocculus and the NRTP afferents mainly terminate in the OMV region. On the efferent side, the cerebellar output to the abducens nucleus from the flocculus/paraflocculus relays through the MVN while the projections related to pursuit from the OMV/FOR, are not known (Ilg and Thier, 2008).

Before leaving the FOR and OMV, one must ask again why there are multiple areas within the cerebellum that seem related to pursuit and saccades. Both pursuit and saccades are commonly associated with other motor behaviors (e.g., head movements,

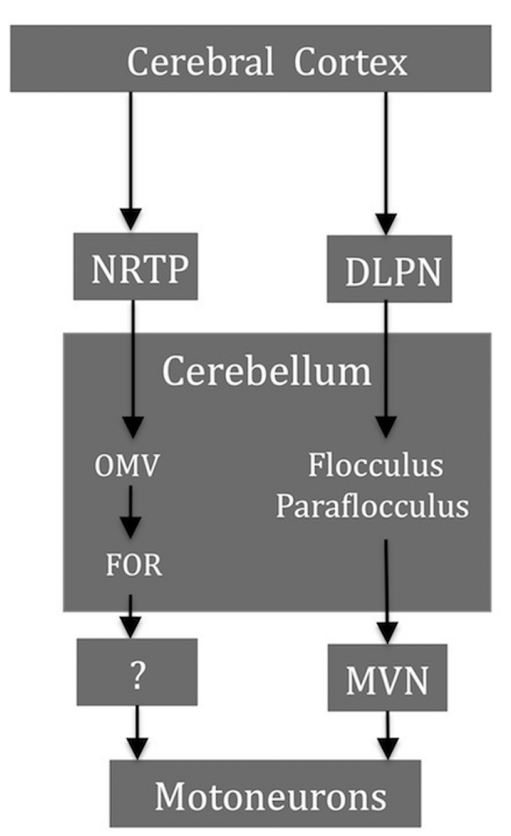

FIGURE 6 | Some smooth pursuit-related structures and their major projections. The brain stem afferents form the DLPN project more strongly to the flocculus/paraflocculus and the NRTP afferents mainly terminate in the OMV region. Cerebellar output from the flocculus/paraflocculus to the ocular motoneurons relays through the MVN whereas the projections from the OMV/FOR are not known. NRTP, nucleus reticularis tegmenti pontis; DLPN, dorsolateral pontine nuclei; OMV, oculomotor vermis; FOR, fastigial oculomotor region; MVN, medial vestibular nucleus. 
arm movements, walking, or running) and also occur in different contexts (e.g., reflexive or self-generated). Thus, one can envision a number of areas within the cerebellum associated with different motor behaviors that might want to use information about associated eye movements to help optimize their own performance.

\section{OTHER ABNORMALITIES OF OCULAR MOTOR CONTROL ASSOCIATED WITH CEREBELLAR DYSFUNCTION ABNORMALITIES OF EYE TORSION}

The torsional orientation of the eye during fixation or movement is precisely controlled and is described by Donders' and Listing's laws. Donders' law states that during steady fixation, the torsional orientation of the eyes is fixed for a given eccentric (horizontal and vertical) eye position. In other words, the eye always assumes the same unique spatial orientation at a final gaze position irrespective of where it came from. Listing's law describes the kinematic features of eye rotations that fix the torsional orientation of the globe for a given horizontal and vertical position of the eyes in the orbit. It states that all eye positions can be reached by a rotation from a given reference position (usually taken as straight-ahead position) about axes that all lie in the same plane (Listing's plane).

Listing's law is not perfectly obeyed in patients with cerebellar lesions. This is partly caused by torsional drifts of the eye that change direction with horizontal gaze eccentricity (intorsional in abduction and extorsional in adduction; Straumann et al., 2000). This finding suggests that the cerebellum is involved in generating a tonic torsional signal that maintains the eyes in Listing's plane against the mechanical forces of the globe. The eye positiondependent torsional drift would then be considered a "passive" violation of Listing's law (determined by the elastic forces on the ocular plant) in the absence of the mapped tonic signal. On the other hand, despite the evidence for abnormal torsion outside of Listing's plane, such a violation did not occur during saccades in patients with cerebellar degeneration (Thurtell et al., 2008).

Unlike saccades or pursuit, ocular torsion during the r-VOR depends considerably less on eye position and does not comply with Listing's law (Misslisch et al., 1994). This torsional movement, however, evolves over time: at the onset of head rotation, the response is largely head-fixed (less dependent on eye position) but later torsion dependent on eye position slowly increases, reaching a plateau when steady-state head velocity is reached (Tian et al., 2006). Moreover, the acceleration of the stimulus affects the kinematics of eye motion; responses to high acceleration head (on body) impulses are more head-fixed than responses to lowacceleration en bloc chair rotations. The fact that the r-VOR is more head-fixed has the theoretical advantage of adequately stabilizing the entire visual image on the retina when the head is rotating.

Patients with cerebellar degeneration show significantly more torsion that is dependent on eye position (i.e., less head-fixed torsion) during the r-VOR (Ignashchenkova et al., 2009). This finding indicates that the cerebellum has a large effect on the evolution over time of torsion that is dependent on eye position during the VOR. It may be that the brain monitors changes in torsional orientation of the eyes during the r-VOR, and depending on the eye position, imposes torsion to prevent the eyes from drifting too far from Listing's plane. The function of the cerebellum might then be to modulate this effect to achieve the compromise between the competing goals of keeping eye positions in Listing's plane and maintaining the stability of images on the retina during head rotation. The evolution of head-fixed to eye position-dependent torsion could reflect the activity in the velocity-to-position ocular motor neural integrator and points to the flocculus/paraflocculus, which is an integral part of the neural integrator circuit (Zee et al., 1981).

\section{THE CEREBELLUM AND BINOCULAR OCULAR MOTOR CONTROL}

Most of the findings relating the cerebellum to ocular motor control have dealt with conjugate eye movements. The cerebellum, however, is also involved in adjusting the relative position of the eyes during fixation (static alignment) and the yoking of the eyes during movement (dynamic alignment). Patients with cerebellar damage sometimes show a skew deviation, a vertical misalignment of the eyes that cannot be attributed to a simple ocular muscle weakness (Keane, 1985; Versino et al., 1996; Zee, 1996; Wiest et al., 2001; Wong and Sharpe, 2005; Brodsky et al., 2006; Colen et al., 2008). The misalignment in patients with cerebellar damage often changes its sense with horizontal eye position - most commonly the abducting eye is higher. The source of the skew may be an imbalance in otolith-ocular reflexes. The otolith organs (utricle and saccule) sense acceleration during linear motion of the head for the t-VOR and during static lateral head tilt for ocular counter roll. Patients with cerebellar skew deviation have reduced and disconjugate counter roll gains that depend on the direction of the head tilt (Wong and Sharpe, 2005). There is also a disconjugate reduction in the gain of the $\mathrm{t}-\mathrm{VOR}$, but without differences between directions of head translation (Schlenker et al., 2009). Since the vestibulocerebellum has an important role in modulation of the t-VOR using information from the otoliths, skew deviation might be due to damage in this part of the cerebellum. The dentate nucleus has been also implicated in cerebellar skew deviation based on MRI/CT lesion analysis (Baier et al., 2008). Patients with cerebellar lesions can also show misalignment of the eyes during the r-VOR and during saccades (Versino et al., 1996; Zee et al., 2002).

An esotropia (the eyes turn inward) also occurs in cerebellar disease and, since the esodeviation is greater at distance it has been attributed to a divergence paralysis (Wiest et al., 2001). Analogous to conjugate smooth pursuit and saccade, vergence eye movements can be classified by their response to disparities produced by stimuli moving slowly (ramp or slow vergence) or abruptly (step or fast vergence). Patients with acute cerebellar lesions can show impaired slow but relatively intact fast vergence. Moreover, divergence, but not convergence, can be affected particularly with the lesions in the OMV (Sander et al., 2009). Patients with vestibulocerebellar lesions may also show a divergence-beating nystagmus (convergent slow phases with divergent quick phases; Yee et al., 1979). These abnormalities hint at an excess of convergence tone with some cerebellar lesions.

In monkeys the majority of OMV Purkinje cells that discharge with pursuit also modulate their activity during vergence, and especially convergence (Nitta et al., 2008a). Temporary inactivation of the OMV results in convergence failure (Nitta et al., 
2008b). A similar effect, however, has not been produced after permanent ablation of the OMV in monkeys (Takagi et al., 2003). Rather there is an esodeviation that can also be seen in patients with cerebellar degeneration, presumably reflecting an increase in convergence tone (Gamlin and Zhang, 1996; Ignashchenkova et al., 2009). These findings might be related to sparing of the FOR as inactivation of these deep cerebellar nuclei causes convergence deficits with an exodeviation (Gamlin and Zhang, 1996; Scheurer et al., 2001). The posterior IP, flocculus, and cerebellar hemispheres are also implicated in the control of vergence eye movement. The flocculus also has neurons that discharge in relation to the vergence angle (Miles et al., 1980; Richter et al., 2004). The floccular/IP pathway may be involved in the control of divergence eye movements while the dorsal vermis/FOR pathway may be more related to convergence, but this compartmentalization of functions is hypothetical (Mays et al., 1986; Nitta et al., 2008b). Functional imaging studies in humans also show activation of the cerebellar hemispheres during convergence. Much like pursuit, multiple regions in the cerebellum may play a role in controlling the conjugacy of eye movements and both dynamic and static vergence.

\section{OCULAR MOTOR LEARNING AND THE CEREBELLUM}

Perhaps the most intriguing aspect of cerebellar function is its role in motor learning. The flocculus/paraflocculus has a clear role in ocular motor learning including the amplitude (gain) and the direction (relative to head motion) of the VOR, the pulse-step ratio for saccades, and the gain of smooth pursuit (Takemori and Cohen, 1974; Optican and Robinson, 1980; Zee et al., 1981; Lisberger et al., 1984; Rambold et al., 2002; Medina and Lisberger, 2009). The OMV/FOR, too, has a role in ocular motor learning, especially related to saccades (Golla et al., 2008; Prsa and Thier, 2011). Transient bilateral inactivation of the FOR impairs the relay of adapted signals to the brain stem circuits that generate premotor commands for saccades, but it does not stop all adaptation from occurring (Robinson et al., 2002). This finding suggests the adaptation takes place upstream in the cerebellar cortex (i.e., in the OMV). The OMV is especially involved in modification of the initial acceleration of pursuit and the amplitude of saccades (Takagi et al., 1998, 2000). Temporary inactivation of the OMV with muscimol injections, a GABA agonist, inhibits adaptive lengthening, but has relatively little effect on adaptive shortening of saccade amplitudes. In contrast, injections of bicuculline, a GABA antagonist, impairs adaptive shortening, and facilitates adaptive lengthening of saccade amplitudes. These findings suggest that OMV activity is necessary for amplitude lengthening adaptation of saccades, whereas amplitude shortening adaptation may involve the inhibitory circuits within the OMV (Kojima et al., 2011).

Recent studies have also implicated the cerebellar hemispheres in ocular motor learning. For example, fMRI studies show that the hemispheric lobules VIII-X are active in processing of saccadic errors (van Broekhoven et al., 2009), and TMS over the hemispheric lobule Crus I has a dual effect on saccadic plasticity; potentiating adaptive lengthening and depressing adaptive shortening of saccade amplitudes (Panouilleres et al., 2011). There is also evidence for involvement of the cerebellar hemispheres in adaptation for more voluntary, internally generated saccades such as memory-guided saccades, as opposed to the OMV involvement in more reflexive, visually guided saccades (Nitschke et al., 2004; Alahyane et al., 2008; Kojima et al., 2010b). Damage to the cerebellar cortex appears to affect a fast component of the adaptation process (seconds) more than a slower component that emerges over minutes (Xu-Wilson et al., 2009). There must be even longer timescales of learning that develop with practice over days and weeks. The inability to perform a sequence of movements which depends upon the cerebellum can be a factor in the defective adaptation process. Cerebellar patients have difficulty making a sequence of visually guided saccades. When presented with random, two-step target jumps these patients failed to make a saccade to the first target jump suggesting an inability to carry out parallel processing of saccades (King et al., 2011).

A memory trace for adaptation of the VOR appears to be acquired initially in the cerebellar cortex (i.e., flocculus) whereas the memory for long-term VOR adaptation likely resides outside of the cerebellum, perhaps in the vestibular nuclei (Galiana, 1986; Peterson et al., 1991; Raymond et al., 1996; Anzai et al., 2010). Recall, however, that the cerebellar recipient zones of the vestibular nuclei can be considered a displaced deep cerebellar nuclei. The VOR adaptation for both gain-up and gain-down learning is more effective at lower frequencies (below $4 \mathrm{~Hz}$ ) than higher frequencies (Raymond and Lisberger, 1996; Broussard et al., 1999). When passive rotation is used to induce changes in the VOR gain, the learned change in gain (whether an increase or a decrease) is greatest at the frequency of head rotation and is more pronounced for gainup than for gain-down learning (Robinson, 1976; Lisberger et al., 1983; Raymond and Lisberger, 1996; de Zeeuw et al., 1998; Kimpo et al., 2005; Titley et al., 2009). This selectivity of learning has led to the idea that VOR signals are segregated into frequency "channels" or adaptive filters and the cerebellum may process these channels selectively (Lisberger et al., 1983; Dean et al., 2010; Broussard et al., 2011).

The cerebellum also influences learning associated with disconjugate eye movements. Phoria adaptation to vertical and horizontal disparities can be impaired in patients with cerebellar dysfunction (Milder and Reinecke, 1983; Kono et al., 2002). Information from animal studies is limited in this regard. Monkeys with lesions of the dorsal vermis show a variable deficit in phoria adaptation but those with flocculus/paraflocculus lesions can still adapt to a horizontal disparity (Judge, 1987; Takagi et al., 2003). The deep cerebellar nuclei (FOR or interposed nuclei) are also possible candidates influencing phoria adaptation.

The effect of experimental lesions within the cerebellum on ocular motor function often varies from animal to animal. While differences in the extent of lesions may explain some of this variability, another interpretation relates to the central role of the cerebellum in long-term ocular motor learning. In this formulation, the cerebellum acts to compensate for differences in motor performance that are idiosyncratic from animal to animal, depending upon the particular genetic makeup of each animal, its "life history" of disease and trauma, and the interaction between inherent capabilities and environmental influences during development and aging. The cerebellar mechanisms involved in different aspects of ocular motor learning are a major focus of contemporary neuroscience. 


\section{REFERENCES}

Alahyane, N., Fonteille, V., Urquizar, C., Salemme, R., Nighoghossian, N., Pelisson, D., and Tilikete, C. (2008). Separate neural substrates in the human cerebellum for sensorymotor adaptation of reactive and of scanning voluntary saccades. Cerebellum 7, 595-601.

Alvina, K., and Khodakhah, K. (2010). The therapeutic mode of action of 4aminopyridine in cerebellar ataxia. $J$. Neurosci. 30, 7258-7268.

Angelaki, D. E., and Hess, B. J. (1994). The cerebellar nodulus and ventral uvula control the torsional vestibulo-ocular reflex. J. Neurophysiol. 72, 1443-1447.

Anzai, M., Kitazawa, H., and Nagao, S. (2010). Effects of reversible pharmacological shutdown of cerebellar flocculus on the memory of long-term horizontal vestibuloocular reflex adaptation in monkeys. Neurosci. Res. 68, 191-198.

Baier, B., Bense, S., and Dieterich, M. (2008). Are signs of ocular tilt reaction in patients with cerebellar lesions mediated by the dentate nucleus? Brain 131, 1445-1454.

Baier, B., Stoeter, P., and Dieterich, M. (2009). Anatomical correlates of ocular motor deficits in cerebellar lesions. Brain 132, 2114-2124.

Baloh, R. W., Yue, Q., and Demer, J. L. (1995). The linear vestibuloocular reflex in normal subjects and patients with vestibular and cerebellar lesions. J. Vestib. Res. 5, 349-361.

Barash, S., Melikyan, A., Sivakov, A., Zhang, M., Glickstein, M., and Thier, P. (1999). Saccadic dysmetria and adaptation after lesions of the cerebellar cortex. J. Neurosci. 19, 10931-10939.

Belton, T., and McCrea, R. A. (1999). Contribution of the cerebellar flocculus to gaze control during active head movements. J. Neurophysiol. 81, 3105-3109.

Belton, T., and McCrea, R. A. (2000). Role of the cerebellar flocculus region in the coordination of eye and head movements during gaze pursuit. J. Neurophysiol. 84, 1614-1626.

Belton, T., and McCrea, R. A. (2002). Role of the cerebellar flocculus region in cancellation of the VOR during passive whole body rotation. J. Neurophysiol. 84, 1599-1613.

Belton, T., and McCrea, R. A. (2004). Context contingent signal processing in the cerebellar flocculus and ventral paraflocculus during gaze saccades. J. Neurophysiol. 92, 797-807.
Brodsky, M. C., Donahue, S. P., Vaphiades, M., and Brandt, T. (2006). Skew deviation revisited. Surv. Ophthalmol. 51, 105-128.

Broussard, D. M., Bhatia, J. K., and Hong, J. A. (1999). The dynamics of the vestibulo-ocular reflex after peripheral vestibular damage. II. Comparison with dynamics after optically-induced learning. Exp. Brain Res. 125, 365-374.

Broussard, D. M., Titley, H. K., Antflick, J., and Hampson, D. R. (2011). Motor learning in the VOR: the cerebellar component. Exp. Brain Res. 210, 451-463.

Buttner, U., Straube, A., and Spuler, A. (1994). Saccadic dysmetria and "intact" smooth pursuit eye movements after bilateral deep cerebellar nuclei lesions. J. Neurol. Neurosurg. Psychiatr. 57, 832-834.

Cohen, B., Helwig, D., and Raphan, T. (1987). Baclofen and velocity storage: a model of the effects of the drug on the vestibulo-ocular reflex in the rhesus monkey. J. Physiol.393, 703-725.

Cohen, B., John, P., Yakushin, S. B. Buettner-Ennever, J., and Raphan, T. (2002). The nodulus and uvula: source of cerebellar control of spatial orientation of the angular vestibuloocular reflex. Ann. N. Y. Acad. Sci. 978, 28-45.

Cohen, H., Cohen, B., Raphan, T., and Waespe, W. (1992). Habituation and adaptation of the vestibuloocular reflex: a model of differential control by the vestibulocerebellum. Exp. Brain Res. 90, 526-538.

Colen, C., Ketko, A., George, E., and Van Stavern, G. (2008). Periodic alternating nystagmus and periodic alternating skew deviation in spinocerebellar ataxia type 6. J. Neuroophthalmol. 28, 287-288.

Colnaghi, S., Ramat, S., D’Angelo, E. Cortese, A., Beltrami, G., Moglia, A., and Versino, M. (2011). Theta-burst stimulation of the cerebellum interferes with internal representations of sensory-motor information related to eye movements in humans. Cerebellum doi: 10.1007/s12311-0110282-281. [Epub ahead of print].

de Zeeuw, C. I., Hansel, C., Bian, F., Koekkoek, S. K., van Alphen, A. M., Linden, D. J., and Oberdick, J. (1998). Expression of a protein kinase $\mathrm{C}$ inhibitor in Purkinje cells blocks cerebellar LTD and adaptation of the vestibulo-ocular reflex. Neuron 20, 495-508.

Dean, P., Porrill, J., Ekerot, C. F., and Jorntell, H. (2010). The cerebellar microcircuit as an adaptive filter: experimental and computational evidence. Nat. Rev. Neurosci. 11, 30-43.

Federighi, P., Cevenini, G., Dotti, M. T., Rosini, F., Pretegiani, E., Federico, A., and Rufa, A. (2011). Differences in saccade dynamics between spinocerebellar ataxia 2 and lateonset cerebellar ataxias. Brain 134, 879-891.

Fuchs, A. F., Robinson, F. R., and Straube, A. (1993). Role of the caudal fastigial nucleus in saccade generation. I. Neuronal discharge pattern. J. Neurophysiol. 170, 1723-1740.

Fuchs, A. F., Robinson, F. R., and Straube, A. (1994). Participation of the caudal fastigial nucleus in smooth-pursuit eye movements. I. Neuronal activity. J. Neurophysiol. 72, 2714-2728.

Fujikado, T., and Noda, H. (1987). Saccadic eye movements evoked by micro-stimulation of lobule VII of the cerebellar vermis of macaque monkeys. J. Physiol. 394, 573-594.

Gad, Y. P., and Anastasio, T. J. (2010) Simulating the shaping of the fastigial deep nuclear saccade command by cerebellar Purkinje cells. Neural. Netw. 23, 789-804.

Galiana, H. L. (1986). A new approach to understanding adaptive visualvestibular interactions in the central nervous system. J. Neurophysiol. 55 349-374.

Gamlin, P. D., and Zhang, H. Y. (1996). Effects of muscimol blockade of the posterior fastigial nucleus on vergence and ocular accommodation in the primate. Abstr. Soc. Neurosci. 22, 1034.

Glasauer, S., Stephan, T., Kalla, R., Marti, S., and Straumann, D. (2009). Up-down asymmetry of cerebellar activation during vertical pursuit eye movements. Cerebellum 8 , 385-388.

Goffart, L., Chen, L. L., and Sparks, D. L. (2003). Saccade dysmetria during functional perturbation of the caudal fastigial nucleus in the monkey. Ann. N. Y. Acad. Sci. 1004, 220-228.

Golla, H., Tziridis, K., Haarmeier, T., Catz, N., Barash, S., and Thier, P. (2008). Reduced saccadic resilience and impaired saccadic adaptation due to cerebellar disease. Eur. J. Neurosci. 27, 132-144.

Hain, T. C., Zee, D. S., and Maria, B. L. (1988). Tilt suppression of vestibulo-ocular reflex in patients with cerebellar lesions. Acta Otolaryngol. 105, 13-20.

Halmagyi, G. M., Rudge, P., Gresty M. A., Leigh, R. J., and Zee, D. S.
(1980). Treatment of periodic alternating nystagmus. Ann. Neurol. 8, 609-611.

Hashimoto, M., and Ohtsuka, K. (1995). Transcranial magnetic stimulation over the posterior cerebellum during visually guided saccades in man. Brain 118, 1185-1193.

Hashimoto, T., Sasaki, O., Yoshida, K., Takei, Y., and Ikeda, S. (2003). Periodic alternating nystagmus and rebound nystagmus in spinocerebellar ataxia type 6. Mov. Disord. 18, 1201-1204.

Hayakawa, Y., Nakajima, T., Takagi, M., Fukuhara, N., and Abe, H (2002). Human cerebellar activation in relation to saccadic eye movements: a functional magnetic resonance imaging study. Ophthalmologica 216, 399-405.

Heinen, S. J., and Keller, E. L. (1996). The function of the cerebellar uvula in monkey during optokinetic and pursuit eye movements: single-unit responses and lesion effects. Exp. Brain Res. 110, 1-14.

Helmchen, C., Gottschalk, S., Sander, T., Trillenberg, P., Rambold, H., and Sprenger, A. (2007). Beneficial effects of 3,4-diaminopyridine on positioning down-beat nystagmus in a circumscribed uvulo-nodular lesion. J. Neurol. 254, 1126-1128.

Helmchen, C., Rambold, H., Sprenger, A., Erdmann, C., and Binkofski, F. (2003). Cerebellar activation in opsoclonus: an fMRI study. Neurology 61, 412-415.

Hufner, K., Stephan, T., Kalla, R. Deutschlander, A., Wagner, J., Holt-mannspotter, M., SchulteAltedorneburg, G., Strupp, M. Brandt, T., and Glasauers, S. (2007). Structural and functional MRIs disclose cerebellar pathologies in idiopathic downbeat nystagmus. Neurology 69, 1128-1135.

Ignashchenkova, A., Dash, S., Dicke, P. W., Haarmeier, T., Glickstein, M. and Thier, P. (2009). Normal spatial attention but impaired saccades and visual motion perception after lesions of the monkey cerebellum. $J$. Neurophysiol. 102, 3156-3168.

Ilg, U. J., and Thier, P. (2008). The neural basis of smooth pursuit eye movements in the rhesus monkey brain. Brain Cogn. 68, 229-240.

Jenkinson, N., and Miall, R. C. (2010). Disruption of saccadic adaptation with repetitive transcranial magnetic stimulation of the posterior cerebellum in humans. Cerebellum 9 , 548-555.

Judge, S. J. (1987). Optically-induced changes in tonic vergence and $\mathrm{AC} / \mathrm{A}$ ratio in normal monkeys and 
monkeys with lesions of the flocculus and ventral paraflocculus. Exp. Brain Res. 66, 1-9.

Kalla, R., Deutschlander, A., Hufner, K., Stephan, T., Jahn, K., Glasauer, S., Brandt, T., and Strupp, M. (2006). Detection of floccular hypometabolism in downbeat nystagmus by fMRI. Neurology 66, 281-283.

Kalla, R., Glasauer, S., Buttner, U., Brandt, T., and Strupp, M. (2007). 4aminopyridine restores vertical and horizontal neural integrator function in downbeat nystagmus. Brain 130, 2441-2451.

Keane, J. R. (1985). Alternating skew deviation: 47 patients. Neurology 35, 725-728.

Kettner, R. E., Suh, M., Davis, D., and Leung, H. C. (2002). Complex predictive eye pursuit in monkey: a model system for cerebellar studies of skilled movement. Arch. Ital. Biol. 140, 331-340.

Kim, J. S., Ahn, K. W., Moon, S. Y., Choi, K. D., Park, S. H., and Koo, J. W. (2005). Isolated perverted head-shaking nystagmus in focal cerebellar infarction. Neurology 64, 575-576.

Kimpo, R., Boyden, E. S., Katoh, A., Ke, M. C., and Raymond, J. L. (2005). Distinct patterns of stimulus generalization of increases and decreases in VOR gain. J. Neurophysiol. 94, 3092-3100.

King, S., Chen, A. L., Joshi, A., Serra, A., and Leigh, R. J. (2011). Effects of cerebellar disease on sequences of rapid eye movements. Vision Res. 51, 1064-1074.

Kojima, Y., Soetedjo, R., and Fuchs, A. F. (2010a). Effects of GABA agonist and antagonist injections into the oculomotor vermis on horizontal saccades. Brain Res. 1366, 93-100.

Kojima, Y., Soetedjo, R., and Fuchs, A. F. (2010b). Behavior of the oculomotor vermis for five different types of saccade. J. Neurophysiol. 104, 3667-3676.

Kojima, Y., Soetedjo, R., and Fuchs, A. F. (2011). Effect of inactivation and disinhibition of the oculomotor vermis on saccade adaptation. Brain Res. 1401, 30-39.

Kono, R., Hasebe, S., Ohtsuki, H., Kashihara, K., and Shiro, Y. (2002). Impaired vertical phoria adaptation in patients with cerebellar dysfunction. Invest. Ophthalmol. Vis. Sci. 43, 673-678.

Krauzlis, R. J., and Miles, F. A. (1998). Role of the oculomotor vermis in generating pursuit and saccades: effects of microstimulation. $\mathrm{J}$. Neurophysiol. 80, 2046-2062.
Leigh, R. J., Das, V. E., and Seidmann, S. H. (2002). A neurobiological approach to acquired nystagmus. Ann. N. Y. Acad. Sci. 956, 380-390.

Leigh, R. J., Robinson, D. A., and Zee, D. S. (1981). A hypothetical explanation for periodic alternating nystagmus: instability in the optokineticvestibular system. Ann. N. Y. Acad. Sci. 374, 619-635.

Leigh, R. J., and Zee, D. S. (2006). The Neurology of Eye Movements. New York: Oxford University.

Lin, C. Y., and Young, Y. H. (1999). Clinical significance of rebound nystagmus. Laryngoscope 109, 1803-1805.

Lisberger, S. G., Miles, F. A., and Optican, L. M. (1983). Frequencyselective adaptation: evidence for channels in the vestibulo-ocular reflex? J. Neurosci. 3, 1234-1244.

Lisberger, S. G., Miles, F. A., and Zee, D. S. (1984). Signals used to compute errors in monkey vestibuloocular reflex: possible role of flocculus. J. Neurophysiol. 52, 1140-1153.

Marti, S., Straumann, D., Buttner, U., and Glasauer, S. (2008). A modelbased theory on the origin of downbeat nystagmus. Exp. Brain Res. 188, 613-631.

Marti, S., Straumann, D., and Glasauer, S. (2005). The origin of downbeat nystagmus: an asymmetry in the distribution of on-directions of vertical gaze-velocity Purkinje cells. Ann. N. Y. Acad. Sci. 1039, 548-553.

Mays, L. E., Porter, J. D., Gamlin, P. D., and Tello, C. A. (1986). Neural control of vergence eye movements: neurons encoding vergence velocity. J. Neurophysiol. 56, 1007-1021.

Medina, J. F., and Lisberger, S. G. (2009). Encoding and decoding of learned smooth-pursuit eye movements in the floccular complex of the monkey cerebellum. J. Neurophysiol. 102, 2039-2054.

Milder, D. G., and Reinecke, R. D. (1983). Phoria adaptation to prisms: a cerebellar dependent response. Arch. Neurol. 40, 339-342.

Miles, F. A., Fuller, J. H., Braitman, D. J., and Dow, B. M. (1980). Long-term adaptive changes in primate vestibuloocular reflex. III. Electrophysiological observations in flocculus of normal monkeys. J. Neurophysiol. 43, 1437-1476.

Misslisch, H., Tweed, D., Fetter, M., Sievering, D., and Koenig, E. (1994). Rotational kinematics of the human vestibuloocular reflex. III. Listing's law. J. Neurophysiol. 72, 2490-2502.

Moon, I. S., Kim, J. S., Choi, K. D., Kim, M. J., Oh, S. Y., Lee, H., Lee, H. S., and Park, S. H. (2009). Isolated nodular infarction. Stroke 40 , 487-491.

Nitschke, M. F., Binkofski, F., Buccino, G., Posse, S., Erdmann, C. Kompf, D., Seitz, R. J., and Heide, W. (2004). Activation of cerebellar hemispheres in spatial memorization of saccadic eye movements: an fMRI study. Hum. Brain Mapp. 22, 155-164.

Nitta, T., Akao, T., Kurkin, S., and Fukushima, K. (2008a). Vergence eye movement signals in the cerebellar dorsal vermis. Prog. Brain Res. 171, 173-176.

Nitta, T., Akao, T., Kurkin, S., and Fukushima, K. (2008b). Involvement of the cerebellar dorsal vermis in vergence eye movements in monkeys. Cereb. Cortex 18, 1042-1057.

Noda, H., and Fujikado, T. (1987) Involvement of Purkinje cells in evoking saccadic eye movements by microstimulation of the posterior cerebellar vermis of monkeys. J. Neurophysiol. 57, 1247-1261.

Noda, H., Sugita, S., and Ikeda, Y (1990). Afferent and efferent connections of the oculomotor region of the fastigial nucleus in the macaque monkey. J. Comp. Neurol. 302, 330-348.

Ohki, M., Kitazawa, H., Hiramatsu, T., Kaga, K., Kitamura, T., Yamada, J., and Nagao, S. (2009). Role of primate cerebellar hemisphere in voluntary eye movement control revealed by lesion effects. J. Neurophysiol. 101, 934-937.

Ohtsuka, K., and Enoki, T. (1998). Transcranial magnetic stimulation over the posterior cerebellum during smooth pursuit eye movements in man. Brain 121, 429-435.

Ohtsuka, K., and Noda, H. (1991). Saccadic burst neurons in the oculomotor region of the fastigial nucleus of macaque monkeys. J. Neurophysiol. 65, 1422-1434.

Optican, L. M., and Robinson, D. A. (1980). Cerebellar-dependent adaptive control of primate saccadic system. J. Neurophysiol. 44, 1058-1076.

Panouilleres, M., Neggers, S. F., Gutteling, T. P., Salemme, R., Stigchel, S. V., van der Geest, J. N., Frens, M. A., and Pelisson, D. (2011). Transcranial magnetic stimulation and motor plasticity in human lateral cerebellum: dual effect on saccadic adaptation. Hum Brain Mapp. doi: 10.1002/hbm.21301. [Epub ahead of print].

Peterson, B. W., Baker, J. F., and Houk, J. C. (1991). A model of adaptive control of vestibuloocular reflex based on properties of cross-axis adaptation. Ann. N. Y. Acad. Sci.627, 319-337.

Prsa, M., and Thier, P. (2011). The role of the cerebellum in saccadic adaptation as a window into neural mechanisms of motor learning. Eur. J. Neurosci. 33, 2114-2128.

Pula, J. H., Gomez, C. M., and Kattah, J. C. (2010). Ophthalmologic features of the common spinocerebellar ataxias. Curr. Opin. Ophthalmol. 21, 447-453.

Quaia, C., Lefevre, P., and Optican, L. M. (1999). Model of the control of saccades by superior colliculus and cerebellum. J. Neurophysiol. 82, 999-1018.

Rambold, H., Churchland, A., Selig, Y., Jasmin, L., and Lisberger, S. G. (2002). Partial ablations of the flocculus and ventral paraflocculus in monkeys cause linked deficits in smooth pursuit eye movements and adaptive modification of the VOR. J. Neurophysiol. 87, 912-924.

Raymond, J. L., and Lisberger, S. G. (1996). Behavioral analysis of signals that guide learned changes in the amplitude and dynamics of the vestibulo-ocular reflex. J. Neurosci. 16, 7791-7802.

Raymond, J. L., Lisberger, S. G., and Mauk, M. D. (1996). The cerebellum: a neuronal learning machine? Science 272, 1126-1131.

Richter, H. O., Costello, P., Sponheim, S. R., Lee, J. T., and Pardo, J. V. (2004). Functional neuroanatomy of the human near/far response to blur cues: eye-lens accommodation/vergence to point targets varying in depth. Eur. J. Neurosci. 20, 2722-2732.

Robinson, D. A. (1976). Adaptive gain control of vestibuloocular reflex by the cerebellum. J. Neurophysiol. 39, 954-969.

Robinson, F. R. (2000). Role of the cerebellar posterior interpositus nucleus in saccades I. Effect of temporary lesions. J. Neurophysiol. 84, 1289-1302.

Robinson, F. R., Fuchs, A., and Noto, C. (2002). Cerebellar influences on saccade plasticity. Ann. N. Y. Acad. Sci. 956, 155-163.

Robinson, F. R., Straube, A., and Fuchs, A. F. (1993). Role of the caudal fastigial nucleus in saccade generation. II. Effects of muscimol inactivation. J. Neurophysiol. 70, 1741-1758.

Robinson, F. R., Straube, A., and Fuchs, A. F. (1997). Participation of caudal fastigial nucleus in smooth pursuit eye movements. II. Effects of muscimol inactivation. J. Neurophysiol. 78, 848-859. 
Sander, T., Sprenger, A., Neumann, G., Machner, B., Gottschalk, S., Rambold, H., and Helmchen, C. (2009). Vergence deficits in patients with cerebellar lesions. Brain 132, 103-115.

Sato, H., and Noda, H. (1992). Posterior vermal Purkinje cells in macaques responding during saccades, smooth pursuit, chair rotation and/or optokinetic stimulation. Neurosci. Res. 12, 583-595.

Scheurer, W., Petz, T., Eggert, T., and Straube, A. (2001). Static alignment after unilateral and bilateral pharmacological inactivation of the caudal fastigial nucleus in the monkey. Abstr. Soc. Neurosci. 27.

Schlenker, M., Mirabella, G., Goltz, H. C., Kessler, P., Blakeman, A. W., and Wong, A. M. (2009). The linear vestibulo-ocular reflex in patients with skew deviation. Invest. Ophthalmol. Vis. Sci. 50, 168-174.

Serra, A., Liao, K., Martinez-Conde, S., Optican, L. M., and Leigh, R. J. (2008). Suppression of saccadic intrusions in hereditary ataxia by memantine. Neurology 70, 810-812.

Shaikh, A. G., Ghasia, F. F., Dickman, J. D., and Angelaki, D. E. (2005). Properties of cerebellar fastigial neurons during translation, rotation, and eye movements. J. Neurophysiol. 93, 853-863.

Shaikh, A. G., Marti, S., Tarnutzer, A. A., Palla, A., Crawford, T. O., Straumann, D., Carey, J. P., Nguyen, K., and Zee, D. S. (2011). Ataxia telangiectasia: a "disease model" to understand the cerebellar control of vestibular reflexes. J. Neurophysiol. 105, 3034-3041.

Stone, L. S., and Lisberger, S. G. (1990). Visual responses of Purkinje cells in the cerebellar flocculus during smooth-pursuit eye movements in monkeys. I. Simple spikes. J. Neurophysiol. 63, 1241-1261.

Straube, A., Deubel, H., Ditterich, J., and Eggert, T. (2001). Cerebellar lesions impair rapid saccade amplitude adaptation. Neurolog $y 5$, 2105-2108.

Straube, A., Scheuerer, W., Robinson, F. R., and Eggert, T. (2009). Temporary lesions of the caudal deep cerebellar nucleus in nonhuman primates. Gain, offset, and ocular alignment. Ann. N. Y. Acad. Sci. 1164, 119-126.

Straumann, D., Zee, D. S., and Solomon, D. (2000). Three-dimensional kinematics of ocular drift in humans with cerebellar atrophy. J. Neurophysiol. 83, 1125-1140.
Strupp, M., Schuler, O., Krafczyk, S., Jahn, K., Schautzer, F., Buttner, U., and Brandt, T. (2003). Treatment of downbeat nystagmus with 3,4-diaminopyridine: a placebo-controlled study. Neurology $61,165-170$.

Suzuki, D., and Keller, E. L. (1988). The role of the posterior vermis of monkey cerebellum in smoothpursuit eye movement control. I. Eye and head movement-related activity. J. Neurophysiol. 59, 1-18.

Suzuki, D. A., Noda, H., and Kase, M. (1981). Visual and pursuit eye movement-related activity in posterior vermis of monkey cerebellum. $J$. Neurophysiol. 46, 1120-1139.

Swartz, B. E., Li, S., Bespalova, I., Burmeister, M., Dulaney, E., Robinson, F. R., and Leigh, R. J. (2003). Pathogenesis of clinical signs in recessive ataxia with saccadic intrusions. Ann. Neurol. 54, 824-828.

Takagi, M., Tamargo, R., and Zee, D. S. (2003). Effects of lesions of the cerebellar oculomotor vermis on eye movements in primate: binocular control. Prog. Brain Res. 142, 19-33.

Takagi, M., Zee, D. S., and Tamargo, R. J. (1998). Effects of lesions of the oculomotor vermis on eye movements in primate: saccades. J. Neurophysiol. 80, 1911-1931.

Takagi, M., Zee, D. S., and Tamargo, R. J. (2000). Effects of lesions of the oculomotor cerebellar vermis on eye movements in primate: smooth pursuit. J. Neurophysiol. 83, 2047-2062.

Takemori, S., and Cohen, B. (1974). Loss of visual suppression of vestibular nystagmus after flocculus lesions. Brain Res. 72, 213-224.

Thurtell, M. J., Raphan, T., Black, R. A., Todd, M. J., Leigh, R. J., and Halmagyi, G. M. (2008). Threedimensional kinematics of saccadic eye movements in humans with cerebellar degeneration. Prog. Brain Res. 171, 215-218.

Tian, J., Zee, D. S., and Walker, M. F. (2006). Eye-position dependence of torsional velocity during stepramp pursuit and transient yaw rotation in humans. Exp. Brain Res. 171, 225-230.

Titley, H. K., Heskin-Sweezie, R., and Broussard, D. M. (2009). Consolidation and disruption of motor memory generalize across stimulus conditions in the vestibulo-ocular reflex. Brain Res. 1267, 37-43.

van Broekhoven, P. C., Schraa-Tam, C. K., van der Lugt, A., Smits, M., Frens, M. A., and van der Geest, J. N. (2009). Cerebellar contributions to the processing of saccadic errors. Cerebellum 8, 403-415.

Velazquez-Perez, L., Seifried, C. Abele, M., Rodriguez-Labrada, R., Santos-Falcon, N., Sanchez-Cruz, G., Almaguer-Mederos, L., Tejeda, R., Canales-Ochoa, N., Fetter, M., Ziemann, U., Klockgether, T. Medrano-Montero, J., RodriguezDiaz, J., Laffita-Mesa, J. M., and Auburger, G. (2009). Saccade velocity is reduced in presymptomatic spinocerebellar ataxia type 2. Clin. Neurophysiol. 120, 632-635.

Versino, M., Hurko, O., and Zee, D. S. (1996). Disorders of binocular control of eye movements in patients with cerebellar dysfunction. Brain 119, 1933-1950.

Waespe, W., Cohen, B., and Raphan, T. (1985). Dynamic modification of the vestibulo-ocular reflex by the nodulus and uvula. Science 228, 199-202.

Waespe, W., and Wichmann, W. (1990). Oculomotor disturbances during visual-vestibular interaction in Wallenberg's lateral medullary syndrome. Brain 113, 821-846.

Walker, M. F., Tian, J., Shan, X., Tamargo, R. J., Ying, H., and Zee, D. S. (2008a). Lesions of the cerebellar nodulus and uvula in monkeys: effect on otolith-ocular reflexes. Prog. Brain Res. 171, 167-172.

Walker, M. F., Tian, J., Shan, X., Tamargo, R. J., Ying, H., and Zee, D. S. (2008b). Lesions of the cerebellar nodulus and uvula impair downward pursuit. J. Neurophysiol. 100, 1813-1823.

Walker, M. F., Tian, J., Shan, X., Tamargo, R. J., Ying, H., and Zee, D. S. (2010). The cerebellar nodulus/uvula integrates otolith signals for the translational vestibulo-ocular reflex. PLoS ONE 5, e13981. doi: 10.1371/journal.pone. 0013981

Walker, M. F., Tian, J., Shan, X., Ying, H., Tamargo, R. J., and Zee, D. S. (2009). Enhancement of the bias component of downbeat nystagmus after lesions of the nodulus and uvula. Ann. N. Y. Acad. Sci. 1164, 482-485.

Walker, M. F., and Zee, D. S. (1999). Directional abnormalities of vestibular and optokinetic responses in cerebellar disease. Ann. N. Y. Acad. Sci. 871, 205-220.

Walker, M. F., and Zee, D. S. (2005a). Cerebellar disease alters the axis of the high-acceleration vestibuloocular reflex. J. Neurophysiol. 94, 3417-3429.

Walker, M. F., and Zee, D. S. (2005b). Asymmetry of the pitch vestibuloocular reflex in patients with cerebellar disease. Ann. N. Y. Acad. Sci. 1039, 349-358.

Wearne, S., Raphan, T., and Cohen, B. (1998). Control of spatial orientation of the angular vestibuloocular reflex by the nodulus and uvula. $J$. Neurophysiol. 79, 2690-2715.

Wiest, G., Tian, J. R., Baloh, R. W., Crane, B. T., and Demer, J. L. (2001). Otolith function in cerebellar ataxia due to mutations in the calcium channel gene CACNA1A. Brain 124, 2407-2416.

Wong, A. M., Musallam, S., Tomlinson, R. D., Shannon, P., and Sharpe, J. A. (2001). Opsoclonus in three dimensions: oculographic, neuropathologic and modeling correlates. J. Neurol. Sci. 189, 71-81.

Wong, A. M., and Sharpe, J. A. (2005). Cerebellar skew deviation and the torsional vestibuloocular reflex. Neurology 65, 412-419.

Xiong, G., Nagao, S., and Kitazawa, H. (2010). Mossy and climbing fiber collateral inputs in monkey cerebellar paraflocculus lobulus petrosus and hemispheric lobule VII and their relevance to oculomotor functions. Neurosci. Lett. 468, 282-286.

Xu-Wilson, M., Chen-Harris, H., Zee, D. S., and Shadmehr, R. (2009). Cerebellar contributions to adaptive control of saccades in humans. $J$. Neurosci. 29, 12930-12939.

Yakusheva, T., Blazquez, P. M., and Angelaki, D. E. (2008). Frequencyselective coding of translation and tilt in macaque cerebellar nodulus and uvula. J. Neurosci. 28, 9997-10009.

Ye, B. S., Kim, Y. D., Nam, H. S., Lee, H. S., Nam, C. M., and Heo, J. H. (2010) Clinical manifestations of cerebellar infarction according to specific lobular involvement. Cerebellum 9 , 571-579.

Yee, R. D., Baloh, R. W., Honrubia, V., Lau, C. G., and Jenkins, H. A. (1979) Slow build-up of optokinetic nystagmus associated with downbeat nystagmus. Invest. Ophthalmol. Vis. Sci. $18,622-629$.

Zee, D. S. (1996). Considerations on the mechanisms of alternating skew deviation in patients with cerebellar lesions. J. Vestib. Res. 6, 395-401.

Zee, D. S. and Walker, M. F. (2009). "Cerebellum and oculomotor control," in Encyclopedia of Neuroscience, ed. L. R. Squire (La Jolla, CA: Elsevier Press), 729-736.

Zee, D. S., Walker, M. F., and Ramat, S. (2002). The cerebellar contribution to eye movements based upon lesions: binocular threeaxis control and the translational 
vestibulo-ocular reflex. Ann. N. Y. Acad. Sci. 956, 178-189.

Zee, D. S., Yamazaki, A., Butler, P. H., and Gucer, G. (1981). Effects of ablation of flocculus and paraflocculus on eye movements in primate. J. Neurophysiol. 46, 878-899.

Zhang, Y., Partsalis, A. M., and Highstein, S. M. (1995). Properties of superior vestibular nucleus flocculus target neurons in the squirrel monkey. I. General properties in comparison with flocculus projecting neurons. J. Neurophysiol. 73, 2261-2278.

Conflict of Interest Statement: The authors declare that the research was conducted in the absence of any commercial or financial relationships that could be construed as a potential conflict of interest.

Received: 05 May 2011; paper pending published: 27 June 2011; accepted: 08 August 2011; published online: 01 September 2011.

Citation: Kheradmand $A$ and Zee DS (2011) Cerebellum and ocular motor control. Front. Neur. 2:53. doi: 10.3389/fneur.2011.00053
This article was submitted to Frontiers in Neuro-otology, a specialty of Frontiers in Neurology.

Copyright $\odot 2011$ Kheradmand and Zee. This is an open-access article subject to a non-exclusive license between the authors and Frontiers Media SA, which permits use, distribution and reproduction in other forums, provided the original authors and source are credited and other Frontiers conditions are complied with. 